\title{
Ataxia enzoótica en ciervo rojo (Cervus elaphus) en Argentina
}

\author{
Enzootic ataxia in red deer (Cervus elaphus) in Argentina \\ J P Soler ${ }^{1 *}$, S B Cseh ${ }^{2}$ \\ ${ }^{1}$ Médico Veterinario. Actividad Privada, Argentina \\ ${ }^{2}$ Lic. Bioquímica. Instituto Nacional de Tecnología Agropecuaria. EEA Balcarce.
}

\begin{abstract}
SUMMARY
Enzootic ataxia is a pathology that causes slow and progressive paralysis of the hind limbs in red deer and has been related to copper $(\mathrm{Cu})$ deficiency. This condition is not seen until deer are about 9 months old. The objective of this paper is to describe a clinical case of enzootic ataxia in red deer kept in captivity in Argentina. The problem started with two pregnant female deer that showed hind limb weakness. One was slaughtered for necropsy. Blood and organ samples were taken, and the latter were kept in formaldehyde at $10 \%$. A necropsy of the foetus was also carried out and a liver sample was taken. Grass and water were analyzed. $\mathrm{Cu}, \mathrm{Fe}, \mathrm{Zn}, \mathrm{Mo}$ and $\mathrm{SO}_{4}$ levels were measured in grass, while total salt, $\mathrm{SO}$, $\mathrm{Ca}, \mathrm{Mg}, \mathrm{Na}$ and $\mathrm{Cl}$ levels were measured in water. Disease prevalence was $0.14 \%$. Liver $\mathrm{Cu}$ values were $14.6 \mathrm{ppm}$ and $337 \mathrm{ppm} \mathrm{DM}$ in the female and foetus respectively. Blood $\mathrm{Cu}$ level in the female was $0.5 \mu \mathrm{g} / \mathrm{ml}$ and haematocrit was $46 \%$. In spinal cord a general medullar myelin sheath loss was found, but it was more severe in dorsal regions of the medulla. Vacuolization of white substance with no inflammatory response was also observed. Although liver $\mathrm{Cu}$ levels in the slaughtered female were lower than those considered as limiting, cupraemia was within the normal range. This is a commonly observed characteristic in $\mathrm{Cu}$ deficiency. $\mathrm{Cu}$ liver values in the foetus were also below the normal range. As this is a cronic disease, it is probable that the animals were exposed to a low $\mathrm{Cu}$ level diet for a long time. This condition could have been worsened by the high $\mathrm{Cu}$ demand during foetal growth in the last third of the gestation period. Since this disease is usually found in certain geographical areas and appears seasonally, it is important to take blood samples at the time of higher risk (beginning of spring) in order to carry out an early diagnosis. Therefore, according to the clinical, laboratory and epidemiological data, it is concluded that the diagnosis corresponds to enzootic ataxia due to $\mathrm{Cu}$ deficiency.
\end{abstract}

Palabras clave: ciervo rojo, ataxia enzoótica, cobre.

Key words: red deer, enzootic ataxia, copper.

\section{INTRODUCCION}

El ciervo rojo (Cervus elaphus) en estado salvaje es un animal poco propenso a contraer enfermedades, aunque esta situación se modifica cuando los ciervos son criados en cautiverio. En estas condiciones pueden aparecer distintas enfermedades, entre las cuales pueden citarse las deficiencias minerales.

La ataxia enzoótica (AE) es una patología de los ciervos que causa parálisis lenta y progresiva en las extremidades posteriores. Es originada por una leucomielopatía del tejido nervioso, caracterizada por una desmielinización de los axones pertenecientes a la médula espinal. Dicha patología está asociada en la mayoría de los casos a una deficiencia de cobre $(\mathrm{Cu})$, siendo considerado este mineral como el principal factor etiológico (Barlow y Butler 1964, Wilson y col 1979, Mc Taggarat y col 1981, Booth y col 1989, Audigé y col 1995).

Aceptado: 23.03.2006.

* E-mail: vetjuansoler@hotmail.com Tel: (0054) 0223-154215373. Dirección: Roca 2650. Dpto 3. Mar del Plata (CP 7600). Provincia de Buenos Aires, Argentina.
$\mathrm{El} \mathrm{Cu}$ es un oligoelemento esencial para el correcto funcionamiento de numerosas enzimas involucradas, entre otras funciones en la síntesis de glóbulos rojos, matriz proteica de los huesos, cartílagos, tendones, melanina y queratina. Es además de gran importancia para el normal desarrollo del sistema nervioso (Kaneko 1989).

El primer diagnóstico de esta enfermedad en la Argentina fue en el año 1995 (Campero y col 1996); sin embargo, la AE ya ha sido ampliamente descrita con anterioridad principalmente en Europa y Nueva Zelanda (Alexander y Buxton 1994).

La morbilidad de la AE suele ser muy baja, usualmente menor a $1 \%$, aunque en raras ocasiones se han observado casos de hasta el 13\% (Thompson y col 1994).

Si bien tiene algunas similitudes con la $\mathrm{AE}$ que se observa en corderos recién nacidos, la principal diferencia radica que en los ciervos rojos sólo se presenta a partir de los 9 meses de edad (Wilson y col 1979).

El objetivo del presente trabajo es describir un caso de $\mathrm{AE}$ en ciervos rojos ocurrido en el año 2002 en un establecimiento que se dedica a la cría de esta especie en Argentina. 


\section{ANTECEDENTES DE MANEJO DE LOS ANIMALES}

El establecimiento en el cual se presentó este caso se encuentra ubicado en el partido de Coronel Suárez $\left(37^{\circ} 28^{\prime}\right.$ latitud sur y $61^{\circ} 56^{\prime}$ longitud oeste), provincia de Buenos Aires, Argentina. Tiene destinadas 350 has a la explotación del ciervo rojo y la finalidad de la cría de esta especie es la producción de carne, velvet, cornamenta dura y la venta de animales destinados a reproducción y caza.

La alimentación se basa, dependiendo de la época del año, en el consumo de pasturas de gramíneas y leguminosas, verdeos de invierno, verdeos de verano, heno de alfalfa y silaje de planta entera de maíz, siendo este último el principal componente de la dieta consumida durante los meses de invierno y principios de primavera.

La existencia de hembras y machos era de 740 y 680 animales, respectivamente. Las edades de los mismos oscilaban entre 12 meses y 7 años tanto para los machos como para las hembras. Del total de hembras, 411 estaban preñadas, hallándose separadas en lotes de 40 ciervas cada uno. El período de servicio abarcó desde marzo hasta mayo, y la parición comenzó a principios de noviembre.

\section{DESCRIPCION DE LOS CASOS Y DISCUSION}

El problema se inició el día 12 de noviembre del 2002, observándose dos ciervas de diferentes lotes con ataxia, caracterizada por debilidad del tren posterior que dificultaba su desplazamiento. Ambos animales adoptaban la posición de perro sentado, permaneciendo gran parte del tiempo echados. Su estado sensorial se veía normal, al igual que la condición corporal. Por no presentar mejoría o evolución de los signos clínicos se procedió al sacrificio de las dos ciervas luego de haber transcurrido tres días desde el comienzo de los mismos.

A una de las hembras se le hizo necropsia y se tomaron muestras de diversos órganos que se conservaron en formol al 10\% (hígado, riñón, bazo, páncreas, glándula adrenal, intestino delgado y grueso, abomaso, pulmón, hemisferio cerebral, médula cervical, médula torácica, médula lumbar, músculo cardíaco y músculo esquelético), suero y sangre entera. Al feto también se le hizo necropsia y se le tomó una muestra de hígado. En los especímenes obtenidos se realizaron estudios histopatológicos, determinación de los valores de Cu en suero sanguíneo, en hígado fetal y materno, y hematocrito en sangre entera.

Del potrero donde se encontraba el animal afectado se tomaron muestras de pasto y agua. En el pasto se cuantificaron los niveles de $\mathrm{Cu}$, hierro $(\mathrm{Fe})$ y zinc $(\mathrm{Zn})$ por espectrofotometría de absorción atómica (EAA), (Perkin Elmer 1982), molibdeno (Mo), (Bingley 1959) y sulfatos $\left(\mathrm{SO}_{4}\right)$, (Cseh y col 1993). En el agua se analizaron los valores de sales totales (ST) por gravimetría: $\mathrm{SO}_{4}$, calcio
$(\mathrm{Ca})$, magnesio $(\mathrm{Mg})$, sodio $(\mathrm{Na})$ por EAA, cloruros $(\mathrm{Cl})$, (Kit comercial de Merck) y pH.

Todas las muestras tomadas fueron remitidas para su análisis a los respectivos laboratorios pertenecientes a la Estación Experimental Agropecuaria del INTA Balcarce.

Ante la sospecha de una deficiencia de $\mathrm{Cu}$ se procedió a suplementar a los animales por vía oral con $95 \mathrm{mg}$ $\mathrm{Cu} /$ cabeza/día.

La prevalencia de esta enfermedad fue del $0,14 \%$, considerándose el total de los animales (1420).

En la necropsia de la hembra afectada y de su feto no se observaron lesiones aparentes.

Los valores de $\mathrm{Cu}$ hepático hallados en la hembra y su feto fueron 14,6 ppm y $337 \mathrm{ppm}$ en materia seca (MS), respectivamente. La concentración de $\mathrm{Cu}$ en sangre de la cierva fue de $0,5 \mu \mathrm{g} / \mathrm{ml}$. El resultado obtenido del hematocrito fue $46 \%$. En la médula espinal torácica, cervical y lumbar, se encontró tumefacción y degeneración mielínica generalizada con pérdida de la vaina de mielina, siendo de mayor severidad en las regiones dorsales de la médula (figura 1). También se observó pérdida de axones y vacuolización de la sustancia blanca sin respuesta inflamatoria importante, salvo por la presencia de alguna reacción celular histiocitaria leve localizada preferentemente en las zonas afectadas (figura 2). El resto de los órganos no presentó alteraciones histológicas.

De acuerdo a las alteraciones histopatológicas observadas en médula espinal se puede decir que la debilidad del tren posterior que presentaba la cierva fue originada por la lesión nerviosa hallada en el análisis histológico.

En el cuadro 1 se detallan los análisis químicos en pasto y agua.

$\mathrm{Si}$ bien el rol del $\mathrm{Cu}$ en la aparición de la ataxia enzoótica en ciervos adultos no ha sido aún totalmente dilucidado, se sabe que este oligoelemento está relacionado con la presentación de esta patología (Audigé y col 1995).

Numerosas enzimas como la superóxido-dismutasa, 2,3 dioxigenasa, lisina-oxidasa, monoamino-oxidasa, citocromo-oxidasa, dopamina-beta-hidraxilasa y aminolevulinato-hidratasa son $\mathrm{Cu}$ dependientes y tienen fun-

Cuadro 1. Análisis químico de una muestra de forraje y agua obtenido en el diagnóstico de ataxia enzoótica en ciervo rojo.

Chemical analysis of water and grass samples obtained in the diagnosis of enzootic ataxia in red deer.

\begin{tabular}{ccccccc}
\hline \multicolumn{7}{c}{ ANALISIS DE PASTO } \\
\hline $\begin{array}{c}\mathrm{Cu} \\
(\mathrm{ppm})\end{array}$ & $\begin{array}{c}\mathrm{Mo} \\
(\mathrm{ppm})\end{array}$ & $\mathrm{Cu}: \mathrm{Mo}$ & $\begin{array}{c}\mathrm{Zn} \\
(\mathrm{ppm})\end{array}$ & $\begin{array}{c}\mathrm{Fe} \\
\mathrm{Fe}(\mathrm{ppm})\end{array}$ & $\begin{array}{c}\mathrm{SO}_{4} \\
(\%)\end{array}$ \\
\hline 7 & 1,1 & 6,36 & 28 & 163 & 0,14 \\
\hline \multicolumn{7}{c}{ ANALISIS DE AGUA } \\
\hline $\mathrm{pH}$ & $\begin{array}{c}\mathrm{ST} \\
(\mathrm{mg} / \mathrm{l})\end{array}$ & $\begin{array}{c}\mathrm{SO}_{4} \\
(\mathrm{mg} / \mathrm{l})\end{array}$ & $\begin{array}{c}\mathrm{Ca} \\
(\mathrm{mg} / \mathrm{l})\end{array}$ & $\begin{array}{c}\mathrm{Mg} \\
(\mathrm{mg} / \mathrm{l})\end{array}$ & $\begin{array}{c}\mathrm{Cl} \\
(\mathrm{mg} / \mathrm{l})\end{array}$ & $\begin{array}{c}\mathrm{Na} \\
(\mathrm{mg} / \mathrm{l})\end{array}$ \\
7,8 & 268 & 15 & 6 & 5 & 8 & 60 \\
\hline
\end{tabular}




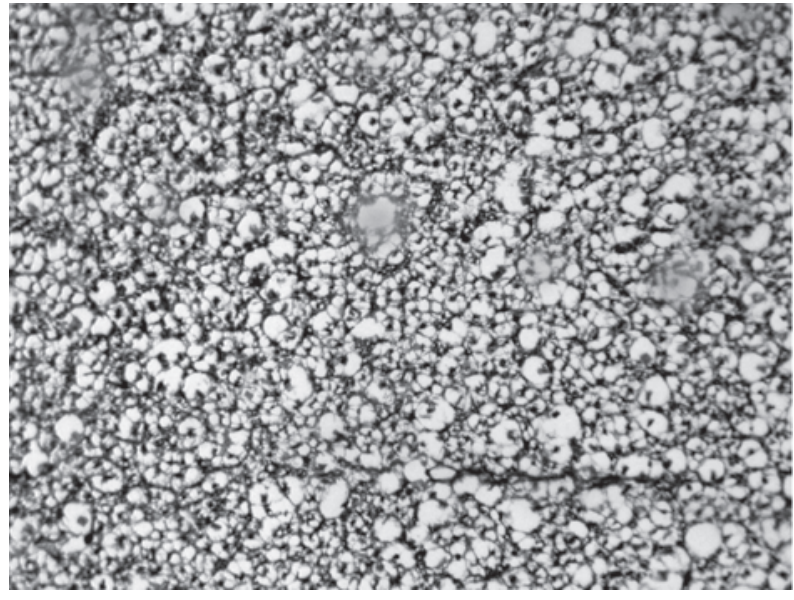

Figura 1. Extensa desmielinización en médula espinal (Luxol fast blue, $\times 100)$ blue, $\times 100)$.

Spinal cord showing extensive demyelination (Luxol fast

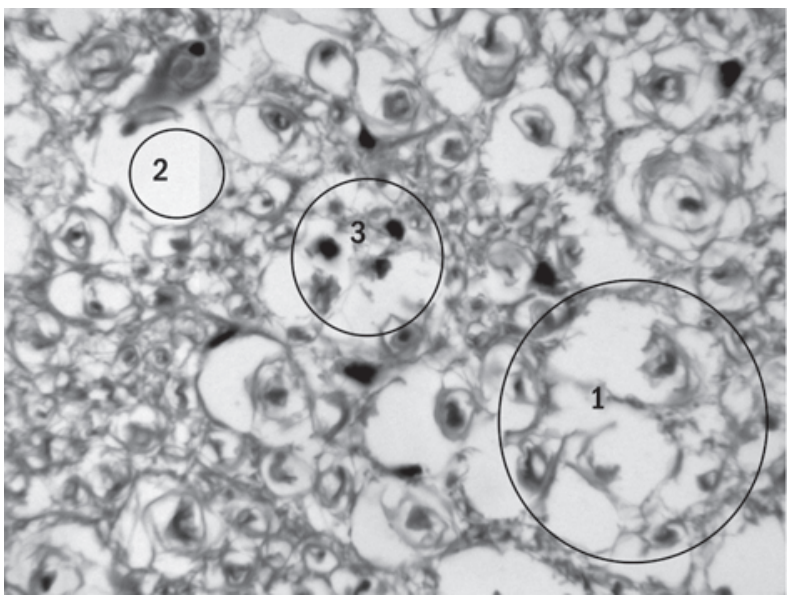

Figura 2. Tumefacción en las vainas de mielina (1) con pérdida de axones (2) y leve reacción celular histiocitaria (3) en médula espinal (Hematoxilina y eosina, $\times 400$ ).

Myelin swelling (1) with loss of axons (2) and mild histiocitic cell reaction (3) in spinal cord (Haematoxylin and eosin, $x$ 400).

ciones relevantes a nivel de sistema nervioso. Así, algunos autores hipotetizan que una deficiencia de Cu podría modificar la actividad de alguna de estas enzimas, originando enfermedades neurodegenerativas (Hartman y Evenson 1992). Otros autores, trabajando con ovinos y caprinos, encontraron que en animales con ataxia del tren posterior existía una alteración en la estructura de la enzima citocromo-oxidasa del cerebro, acompañado esto con una disminución en su actividad enzimática (Alleyne y col 1996, Alleyne y col 1998).

Los niveles hepáticos de $\mathrm{Cu}$ de la hembra afectada se encontraban por debajo del valor considerado como límite (50 ppm MS), (Mackintosh 1998). Diagnósticos de ataxia enzoótica, realizados por diferentes autores, concuerdan en que la totalidad de los animales afectados presentan valores de $\mathrm{Cu}$ hepático por debajo de $25 \mathrm{ppm}$
MS, y la mayor parte de éstos son inferiores a $15 \mathrm{ppm}$ MS (Mac Taggarat y col 1981, Alexander y col 1994, Fyffe 1996).

En este caso, es posible que la demanda adicional de $\mathrm{Cu}$ originada por el crecimiento fetal haya acelerado la depleción del mineral en la madre, lo que sumado a un bajo consumo dietario de este oligoelemento pudo haber precipitado el desarrollo de la AE. Esta hipótesis también fue postulada por Audigé y col (1995), quienes observaron problemas de AE en hembras con avanzado estado de gestación, sugiriendo esto una mayor susceptibilidad de las ciervas preñadas hacia el final del período gestacional.

A pesar de las bajas reservas hepáticas de $\mathrm{Cu}$ que presentaba la hembra necropsiada, la cupremia se hallaba dentro del rango normal $(0,5-1,5 \mu \mathrm{g} / \mathrm{ml})$. Esto se debe a que el organismo es muy eficiente en mantener el nivel de $\mathrm{Cu}$ en sangre dentro de valores óptimos a pesar de los bajas reservas hepáticas (Audigé y col 1995). Esta característica también ha sido observada en otros trabajos donde Wilson y col (1979) y Audigé y col (1995) encontraron que la cupremia en ciervos con ataxia enzoótica fue mayor que en animales no afectados, confirmando esto la necesidad de muestrear no solo los animales con ataxia, sino también los clínicamente normales cuando se desea investigar un probable caso de deficiencia.

Los valores de $\mathrm{Cu}$ hepático fetal encontrados fueron unas 23 veces mayor a los maternos, lo cual es esperable debido a la transferencia placentaria que ocurre durante la gestación que va en detrimento de las reservas maternas. Aun así, este valor de $\mathrm{Cu}$ hepático fetal sigue siendo muy inferior a los valores normales hallados por otros investigadores en ciervos rojos recién nacidos (mayor a 1414 ppm MS), (Reid y col 1980).

Debido a que la AE es una patología de curso crónico y que requiere de un tiempo determinado de deficiencia para alcanzar la etapa de disfunción, es factible pensar que los animales estuvieron expuestos a un consumo dietario de $\mathrm{Cu}$ por debajo de lo recomendado como óptimo durante un periodo prolongado. Este hecho fue observado por Audigé y col (1995), donde encontraron que la cupremia analizada en hembras clínicamente normales se encontraba por debajo del rango normal en el $100 \%$ de las ciervas muestreadas dos meses antes de la aparición de animales con AE.

Teniendo en cuenta lo mencionado anteriormente, hay que destacar que el análisis de pasto y agua realizado en el momento del problema tiene un valor diagnóstico relativo debido a que esta deficiencia suele ser estacional, no sólo por la variación en los requerimientos de los animales durante el año, sino también por las diferencias que existen en la composición mineral de los pastos según la estación. Mackintosh (1998) menciona que los valores de $\mathrm{Cu}$ en el pasto varían a lo largo del año, encontrándose disminuidos en invierno-primavera y tienden a incrementarse hacia el verano-otoño, lo cual se 
correlaciona de la misma manera con los niveles de $\mathrm{Cu}$ en hígado y suero en los animales. Por esta razón es posible que los valores minerales en el alimento se hayan encontrado normales en el momento en que se observó a los animales afectados, siendo difícil saber qué sucedió en los meses anteriores. Para este caso en particular, los resultados indican que en el mes de noviembre los parámetros medidos en pasto y agua estaban dentro de valores óptimos, por lo que no se pudo determinar si la deficiencia fue de tipo primaria o secundaria.

En muchos casos de deficiencias severas de $\mathrm{Cu}$, con o sin presentación de ataxia en animales adultos, se han observado problemas óseos y articulares en bambis de pocos meses de edad, como osteocondrosis y fracturas espontáneas; sin embargo, en nuestro caso no fueron observados dichos problemas en las crías nacidas en esa parición.

Dentro de los diagnósticos diferenciales más importantes se debe mencionar al traumatismo de columna vertebral, tumores que originen compresión medular, meningitis y parasitosis con localización en tejido nervioso (Elaphostrongylus cervi), (Wilson y col 1979).

De acuerdo a los datos clínicos, de laboratorio y epidemiológicos obtenidos de este caso, se concluye que el diagnóstico corresponde a "ataxia enzoótica por deficiencia de $\mathrm{Cu}$ ".

\section{RESUMEN}

Se describe un caso de ataxia enzoótica (AE) en ciervos rojos en cautiverio ocurrido en Argentina. La AE es una patología de los ciervos que causa parálisis lenta y progresiva de las extremidades posteriores y ha sido asociada a la deficiencia de cobre. Su presentación suele ser a partir de los 9 meses de edad. El problema afectó a dos ciervas rojas con debilidad de los miembros posteriores. Se realizó necropsia a una hembra preñada. Se tomaron muestras de órganos en formol al 10\%, de suero y sangre entera. Al feto también se le realizó necropsia y se le tomó una muestra de hígado. A partir de muestras de pasto se determinaron los niveles de $\mathrm{Cu}, \mathrm{Fe}, \mathrm{Zn}, \mathrm{Mo} \mathrm{y} \mathrm{SO}_{4}$. En el agua se analizaron los valores de sales totales, $\mathrm{SO}_{4}, \mathrm{Ca}, \mathrm{Mg}, \mathrm{Na}$ y $\mathrm{Cl}$. $\mathrm{La}$ prevalencia de esta enfermedad en el establecimiento fue del 0,14\%. Los valores de $\mathrm{Cu}$ hepático hallados en la hembra y su feto fueron 14,6 ppm y 337 ppm MS, respectivamente. El nivel de $\mathrm{Cu}$ en sangre de la cierva fue de $0,5 \mu \mathrm{g} / \mathrm{ml}$ y el hematocrito de $46 \%$. En médula espinal se encontró degeneración mielínica generalizada con pérdida de la vaina de mielina, siendo de mayor severidad en las regiones dorsales de la médula. Se observó también vacuolización de la sustancia blanca sin respuesta inflamatoria. Los niveles hepáticos de $\mathrm{Cu}$ en la hembra necropsiada se encontraban por debajo del valor considerado como límite, pero a pesar de esto la cupremia se hallaba dentro del rango de referencia siendo ésta una característica comúnmente observada en los casos de deficiencia de $\mathrm{Cu}$. Los valores de Cu hepático fetal también estaban por debajo del rango considerado como normal. A pesar de que los parámetros medidos en pasto y agua al momento del problema estaban dentro de valores de referencia, es probable que los ciervos hayan estado expuestos previamente a bajos valores de $\mathrm{Cu}$ dietario durante un tiempo prolongado, lo cual pudo verse agravado por la gran demanda de $\mathrm{Cu}$ que ejerce el crecimiento fetal. Considerando que esta enfermedad se presenta usualmente en forma estacional, es de gran importancia realizar muestreos de sangre en la época de mayor riesgo (principios de primavera) con el fin de hacer un diagnóstico temprano. De acuerdo a los datos clínicos, de laboratorio y epidemiológicos, se concluye que el diagnóstico corresponde a "ataxia enzoótica por deficiencia de Cu".

\section{REFERENCIAS}

Alleyne T, A Adogwa, A Lalla, J Joseph, R Jhon. 1996. Novel mitochondrial proteins and decreased intrinsic activity of cytochrome-c-oxidase. Characteristics of swayback disease in sheep. Mol Chem Neuropathol 28, 285-293.

Alleyne T, J Joseph, V Sampson, A Adogwa. 1998. Cytochrome c-oxidase isolated from the brain of swayback disease sheep plays unusual structure and uncharacteristic kinetics. Mol Chem Neuropathol 34, 233-247.

Alexander TL, D Buxton. 1994. Enzootic ataxia. Management and diseases of deer, Second Edition.

Audigé L, PR Wilson, RS Morris, GW Davidson. 1995. Osteochondrosis, skeletal abnormalities and enzootic ataxia associated with copper deficiency in a farmed red deer (Cervus elaphus). $N$ Z Vet J 43, 70-76.

Barlow RM, E J Butler. 1964. An ataxic condition in red deer (Cervus elaphus). J Comp Path 74, 519-529.

Bingley JB. 1959. Simplified determination of molybdenum in plant material by 4-methyl-1-1,2 dimercaptobenzene, Dithiol. J Agr Food Chem 7, 269-270.

Booth DH, PR Wilson, AM Alexander. 1989. The effect of oral copper wire on liver copper in farmed red deer. N Z Vet J 37, 98-101.

Campero CM, R Chayer, S Cseh, M Caracino, G Calderon. 1996. Ataxia en ciervos colorados (Cervus elaphus) por deficiencia de cobre. XX Congreso Argentino de Producción Animal, Río Hondo, Argentina, Pp. 20.

Cseh SB, M Ridao, M Yarrar. 1993. Determinación de sulfatos en agua de bebida. En: IX Reunión Anual de la AAVLD, Tandil, Argentina, Pp 47.

Fyffe J J. 1996. Serum copper concentrations and clinical signs in red deer (Cervus elaphus) during drought in central Victoria. Aust Vet $J$ 73, 188-191.

Hartmann HA, MA Evenson. 1992. Deficiency of copper can cause neuronal degeneration. Med Hypotesis 38, 75-78

Kaneko JJ. 1989. Clinical biochemistry of domestic animals. Fourth edition. Ed. J.J. Kaneko. Academic Press, Inc. California. USA, Pp 932.

Mackintosh CG. 1998. Deer health and disease. Acta Vet Hung 46, 381-394.

Mc Taggarat HS, VPW Lowe, PJ Barden, JBM Gellatly, 1981. Copper status of red deer on the island of Rhum. Vet Rec 109, 155-157.

Perkin Elmer. 1982. Analytical methods for atomic absorption spectrophotometry. Ed. Perkin Elmer Corporation. Connecticut, USA, Pp 530.

Reid TC, HJF Mcallum, PD Johnstone. 1980. Liver copper concentrations in red deer (Cervus elaphus) and wapiti (C. canadiensis) in N. Z. R Vet Sci 28, 261-262.

Thompson KG, L Audigé, DG Arthur, AF Julian, MB Orr, KD Mcsporran, PR Wilson. 1994. Osteochondrosis associated with copper deficiency in young farmed red deer and wapiti-red deer hybrids. $N Z$ Vet $J 42,137-143$.

Wilson PR, B Orr, EL Key. 1979. Enzootic ataxia in Red deer. $N$ Z Vet $J$ 27, 252-254. 